\section{Inferior Parietal Lobule}

\section{Mark Mennemeier}

Neurobiology and Developmental Sciences, University of Arkansas for Medical Sciences, Little Rock, AR, USA

\section{Synonyms}

Inferior parietal area; Inferior parietal cortex; Posterior parietal cortex

\section{Definition}

The IPL is comprised of heteromodal association cortex located on the lateral surface of the brain. It corresponds to Brodmann's areas 39 and 40 and extends from behind the posterior, post central sulcus to the intraparietal sulcus (Caspers et al. 2008). The supramarginal gyrus (BA 40) makes up the anterior part of the IPL, and the angular gyrus (BA 39) makes up the middle and posterior parts. Damage to the IPL and surrounding areas is associated with neuropsychological syndromes, disorders, and deficits including but not limited to, aprosodia, anosognosia, constructional apraxia, and the neglect syndrome following right hemisphere injury; aphasia, ideomotor apraxia, and the Gerstmann's syndrome following left hemisphere injury; and Balint's syndrome following bilateral injury (Zigmond et al. 1999).

\section{Cross-References}

- Anosognosia

- Aphasia

- Aprosodia

- Balints Syndrome

- Constructional Apraxia

- Gerstmann's Syndrome

- Ideomotor Apraxia

- The Neglect Syndrome

\section{References and Readings}

Caspers, S., Eickhoff, S., Geyer, S., Scheperjans, F., Mohlberg, H., Zilles, K., et al. (2008). The human inferior parietal lobule in stereotaxic space. Brain Structure and Function, 212, 481-495.

Zigmond, M., Bloom, F. E., Landis, S. C., Roberts, J. L., \& Squire, L. R. (1999). Fundamental neuroscience. San Diego: Academic Press. 